\title{
A NONLOCAL DIFFUSION EQUATION ARISING IN TERMINALLY ATTACHED POLYMER CHAINS
}

\author{
By \\ Xinfu Chen \\ and \\ Avner Friedman
}

IMA Preprint Series \# 647

June 1990 


\title{
A NONLOCAL DIFFUSION EQUATION ARISING IN TERMINALLY ATTACHED POLYMER CHAINS
}

\author{
XINFU CHEN* AND AVNER FRIEDMAN†
}

\begin{abstract}
We consider a polymer melt in a domain $\Omega$ whereby each polymer chain is attached at one endpoint to a fixed surface $S$ contained in $\partial \Omega$. Denote by $G(x, t ; y)$ the normalized number density of all subchains from $x$ to $y$ of length $t$. Then, according to the selfconsistent mean field theory, $G$ satisfies, for each $y: G_{t}-\nabla^{2} G+\sigma \varphi G=0$ where $\sigma$ is a real parameter, and $\varphi$ is a functional of $G(\cdot, \cdot ; \cdot)$ both nonlocal and nonlinear. We establish existence of $G$ and $C^{\infty}$ regularity of $\varphi$, as a function of $x$.
\end{abstract}

Introduction. This paper is concerned with a heat equation involving a nonlocal, nonlinear term. The equation models the distribution of polymer chains whereby each chain's endpoint is attached to a given surface $S$; the chains are contained in a domain $\Omega$, and $S$ is a part of the boundary $\partial \Omega$ of $\Omega$.

It is customary to consider polymer chains as consisting of polygonal arcs; each side has a fixed length $l$ called Kuhn's segment and is made of several monomers. Adjacent segments are considered to have directions independent of one another. Consequently the polymer chain may be viewed as a random walk with, say, $N$ steps. We consider here the situation where the initial point of each polymer is attached to $S$, and the chain is a random walk which is constrained to be in $\Omega$.

Denote by $G\left(x, j l ; x^{\prime}\right)$ the probability of a subchain which starts at $x^{\prime}$ and ends at $x$ after $j$ segments. $G$ is affected by all the chains in $\Omega$. The self-consistent mean field theory $(S C F)$ due to Dolan and Edwards $(1974,1975)$ derives, after some approximations, the following equation for $G$ :

$$
G_{t}-\Delta G+\sigma \varphi G=0
$$

where

$$
G\left(x, 0 ; x^{\prime}\right)=\delta_{x^{\prime}}(x) \quad\left(\text { the Dirac measure at } x^{\prime}\right),
$$

and $\varphi(x)$ is a potential representing the probability of all the chains passing through $x$; it is given by

$$
\varphi(x)=\frac{\int_{0}^{T} d s \int_{S} G(x, s ; \eta) d S_{\eta} \int_{\Omega} G(\xi, T-s ; x) d \xi}{\int_{S} d S_{\eta} \int_{\Omega} G(\xi, T ; \eta) d \xi} .
$$

*School of Mathematics, University of Minnesota, Minneapolis, MN 55455

†Institute for Mathematics and its Applications, University of Minnesota, Minneapolis, MN 55455. This work is partially supported by the National Science Foundation Grant DMS-86-12880. 
Here $j l$ was replaced by the time variable $t$, and $T=N l$. More precisely, $\Delta G \equiv \Sigma \partial^{2} G / \partial x_{i}^{2}$ should be replaced by $l^{2} \Delta G$ and $\sigma$ is a constant proportional to the excluded volume coefficient as well as to the surface density of the anchored polymers on $S$; see Muthukumar and Ho (1989). However, after non-dimensionalizing $x$ and $t$ we arrive at (0.1)-(0.3) with $\sigma=O(1)$. The parameter $\sigma$, as well as the excluded volume parameter, depend on the temperature $\theta ; \sigma>0$ (good solvent) for $\theta>\theta_{c}$ and $\sigma<0$ (poor solvent) for $\theta<\theta_{c}$. We refer the reader to recent articles by Patel (1989) and Ploehn and Russel (1989) which explain the SCF model and include extensive bibliographies.

If the boundary $\partial \Omega$ is a hard wall then one imposes the absorbing boundary condition

$$
G\left(x, t ; x^{\prime}\right)=0 \quad \text { for } \quad x \in \partial \Omega .
$$

On the other hand if $\partial \Omega$ is partially reflecting and partially absorbing then the appropriate boundary condition is (see de Gennes (1969))

$$
\left(\frac{\partial G}{\partial \nu}+a G\right)\left(x, t ; x^{\prime}\right)=0 \quad \text { for } \quad x \in \partial \Omega, \quad a \geq 0
$$

where $\nu$ is the outward unit normal to $\partial \Omega$.

Numerical work in the one-dimensional case for the absorbing boundary condition is described in Muthukumar and Ho (1989) and Patel (1989); the main interest of the computation is to discover the shape of the potential function $\varphi(x)$.

In the present paper we shall consider the general case where $\Omega$ is 3 - or $n$-dimensional. For definiteness we shall establish all our results for the boundary condition (0.5); the case $(0.4)$ requires rather minor modifications (the definition of $\varphi$ needs to be slightly modified in this case, since $G(\xi, T ; \eta)=0$ if $\eta \in \partial \Omega)$.

In $\S \S 1,2$ we consider the case where $\sigma>0$, and prove the existence of a solution $G$ with $\varphi$ in $C^{\infty}$. The case $\sigma<0$ is considered in $\S 3$.

$\S 1$. Auxiliary estimates. Let $\Omega$ be a bounded domain in $\mathbb{R}^{n}(n \geq 1)$ with $C^{2}$ boundary and let $a(x)$ be a non-negative Hölder continuous function defined on the boundary $\partial \Omega$ of $\Omega$.

Let $\varphi$ be any function defined in $\bar{\Omega}$ and satisfying:

$$
\begin{aligned}
& \varphi \geq 0 \\
& \varphi \in L^{p}(\Omega) \text { for some } 1<p<\infty \\
& \int_{\Omega} \varphi=\sigma T, \sigma>0, T>0 .
\end{aligned}
$$

We denote by $\Omega_{s}$ the set $\Omega \times\{0<t<s\}$. 
Consider the parabolic problem:

$$
\begin{aligned}
& u_{t}-\Delta u+\varphi u=0 \quad \text { in } \quad \Omega_{T}, \\
& u_{\nu}+a(x) u=0 \text { on } \partial \Omega \times(0, T), \\
& u(x, 0)=u_{0}(x), u_{0}(x) \geq 0 .
\end{aligned}
$$

The existence and uniqueness of a weak solution is valid for any measure $u_{0}$.

If $u_{0}(x)=\delta_{x^{\prime}}(x)$ then we set

$$
G\left(x, t ; x^{\prime}\right)=u \quad \text { if } \quad x^{\prime} \in \Omega ;
$$

when $x^{\prime} \in \partial \Omega$ we take $G\left(x, t ; x^{\prime}\right)$ to be the limit of $G(x, t ; y), y \in \Omega, y \rightarrow x^{\prime}$. Let $S$ be a nonempty open portion of $\partial \Omega$, and set

$$
B(x, t)=\int_{S} G(x, t ; \eta) d S_{\eta} .
$$

If $u_{0}(x)=1$ in (1.4) then we denote the solution by $A(x, t)$.

We are interested in studying the functions $A, B$.

Denote by $\Gamma\left(x, t ; x^{\prime}\right)$ the Green function of

$$
\begin{aligned}
& v_{t}-\Delta v=0 \text { in } \Omega_{T}, \\
& v_{\nu}+a(x) v=0 \quad \text { on } \quad \partial \Omega \times(0, T) .
\end{aligned}
$$

By symmetry,

$$
\begin{aligned}
& \Gamma\left(x, t ; x^{\prime}\right)=\Gamma\left(x^{\prime}, t ; x\right), \\
& G\left(x, t ; x^{\prime}\right)=G\left(x^{\prime}, t ; x\right) .
\end{aligned}
$$

If $\varphi \in C^{\alpha}(\bar{\Omega})$, both (1.2) and (1.3) are satisfied in the usual sense. Then, by the maximum principle,

$$
u \geq 0
$$

and

$$
G\left(x, t ; x^{\prime}\right) \leq \Gamma\left(x, t ; x^{\prime}\right) .
$$

By approximation we can establish (1.7), (1.8) also in case $\varphi$ is only in $L^{p}$. Since $\Gamma$ is a bounded function for $t$ uniformly positive, $\Gamma \varphi$ belongs to $L^{p}$ and, by (1.8), also $G \varphi$ belongs to $L^{p}$. It follows that (1.2) is satisfied in the a.e. sense and that $G_{t}$ and $D_{x}^{2} G$ belong to $L_{\mathrm{loc}}^{p}\left(\Omega_{T}\right)$.

In the sequel we shall denote by $C(T)$ various positive constants which depend on $T$ but are otherwise independent of $\varphi$. 
Lemma 1.1. The function $A(x, t)=\int_{\Omega} G(\xi, t ; x) d \xi$ satisfies:

$$
\begin{gathered}
A \leq 1, \\
\iint_{\Omega_{T}} A_{t}^{2}+\int_{\Omega}|\nabla A(x, t)|^{2} d x \leq C(T) .
\end{gathered}
$$

Proof. The estimate (1.9) follows by comparison, since $u \equiv 1$ is a supersolution.

Next, multiplying

$$
A_{t}-\Delta A+\varphi A=0
$$

by $A_{t}$ and integrating over $\Omega_{t}$ we get, after integration by parts,

$$
\iint_{\Omega_{t}} A_{t}^{2}+\iint_{0}\left(-A_{\nu}\right) A_{t}+\iint_{\Omega_{t}} \frac{1}{2} \frac{d}{d t}|\nabla A|^{2}+\iint_{\Omega_{t}} \frac{1}{2} \varphi \frac{d}{d t} A^{2}=0 .
$$

Since $A_{\nu}+a A=0$, we obtain

$$
\begin{array}{r}
\iint_{\Omega_{t}} A_{t}^{2}+\frac{1}{2} \int_{\Omega}|\nabla A(x, t)|^{2} d x+\frac{1}{2} \int_{\partial \Omega} a(x) A^{2}(x, t) d S_{x}+\frac{1}{2} \int_{\Omega} \varphi(x) A^{2}(x, t) d x \\
=\frac{1}{2} \int_{\partial \Omega} a+\frac{1}{2} \int_{\Omega} \varphi=C(T)
\end{array}
$$

and (1.10) follows.

Lemma 1.2. The function

$$
w=\int_{0}^{t} B(x, s) d s
$$

satisfies:

$$
\iint_{\Omega_{T}} w_{t}^{2}+\int_{\Omega}|\nabla w(x, t)|^{2} d x \leq C(T) .
$$

Proof. Multiplying

$$
B_{t}-\Delta B+\varphi B=0
$$

by

$$
\int_{t}^{\tau} B(x, s) d s
$$


and integrating over $\Omega_{\tau}$ we get

$$
\begin{aligned}
\int_{\Omega} d x & \int_{0}^{\tau} B_{t}(x, t)\left(\int_{t}^{\tau} B(x, s) d s\right) d t \\
& -\int_{0}^{\tau} d t \int_{\Omega} \Delta B(x, t)\left(\int_{t}^{\tau} B(x, s) d s\right) d x+\iint_{\Omega_{\tau}} \varphi(x) B(x, t) \int_{t}^{\tau} B(x, s) d s=0 .
\end{aligned}
$$

The first integral is equal to

$$
\begin{aligned}
& \int_{\Omega} d x\left[B(x, t) \int_{t}^{\tau} B(x, s) d s\right]_{0}^{\tau}+\int_{\Omega} d x \int_{0}^{\tau} B^{2}(x, t) d t \\
& =-\int_{S} \int_{0}^{\tau} B(\eta, s) d s d S_{\eta}+\int_{\Omega_{t}} w_{t}^{2},
\end{aligned}
$$

since $B=w_{t}$. The second term on the left-hand side of (1.14) is equal to

$$
\begin{gathered}
\int_{0}^{\tau} d t\left(\int_{\Omega} \nabla B(x, t) \int_{t}^{\tau} \nabla B(x, s) d s\right) d x-\int_{0}^{\tau} d t \int_{\partial \Omega} B_{\nu}(x, t)\left(\int_{t}^{\tau} B(x, s) d s\right) d S_{x} \\
=\frac{1}{2} \int_{\Omega} d x\left|\int_{0}^{\tau} \nabla B(x, s) d s\right|^{2}+\frac{1}{2} \int_{\partial \Omega} a(x)\left(\int_{0}^{\tau} B(x, s) d s\right)^{2} d S_{x},
\end{gathered}
$$

since $B_{\nu}+a B=0$. Finally, the last term on the left-hand side of (1.14) is equal to

$$
-\iint_{\Omega_{\tau}} \frac{1}{2} \varphi \frac{d}{d t}\left(\int_{t}^{\tau} B(x, s) d s\right)^{2}=\frac{1}{2} \int_{\Omega} \varphi(x)\left(\int_{0}^{\tau} B(x, s) d s\right)^{2} .
$$

Combining these expressions, we get from (1.14)

$$
\begin{gathered}
\iint_{\Omega_{\tau}} w_{t}^{2}+\frac{1}{2} \int_{\Omega}|\nabla w(x, \tau)|^{2}+\frac{1}{2} \int_{\Omega} \varphi w^{2}(x, \tau)+\frac{1}{2} \int_{\partial \Omega} a w^{2}(x, \tau) d S_{x} \\
=\int_{S} w(\eta, \tau) d S_{\eta} .
\end{gathered}
$$

The right-hand side is bounded by

$$
\varepsilon \int_{\Omega}\left[w^{2}(x, \tau)+|\nabla w(x, \tau)|^{2}\right]+C_{\varepsilon}
$$


whereas, since $w(x, 0)=0$,

$$
w(x, \tau)=\int_{0}^{\tau} w_{t}(x, t) d t
$$

so that

$$
\int_{\Omega} w^{2}(x, \tau) \leq C(T) \iint_{\Omega_{\tau}} w_{t}^{2}(x, t) .
$$

Choosing $\varepsilon$ small enough we then deduce from (1.15) that (1.13) holds with $C(T)$ depending on $T$ but not on $\varphi$.

Lemma 1.3. The function $B$ satisfies:

$$
B(x, t) \leq \frac{C(T)}{\sqrt{t}} .
$$

Proof. From (1.5), (1.8),

$$
B(x, t) \leq \int_{S} \Gamma(x, t ; y) d S_{y} \leq C \int_{S} \frac{e^{-\frac{|x-y|^{2}}{4 t}}}{t^{n / 2}} d S_{y}
$$

where $C$ is a positive constant; the last inequality is a well known estimate for the Green function $\Gamma$, and follows from the construction of $\Gamma$ in the form $\Gamma=\Gamma_{0}+h$ where $\Gamma_{0}$ is the fundamental solution of the heat operator and $h$ is a "regular" term which makes $\Gamma$ satisfy the boundary condition (1.3).

Suppose first that $S$ is a domain in $y_{n}=0$. Setting $y^{\prime}=\left(y_{1}, \ldots, y_{n-1}\right), x^{\prime}=$ $\left(x_{1}, \ldots, x_{n-1}\right)$, we can estimate the right-hand side of (1.17) by

$$
C \int_{\left|y^{\prime}\right|<C} \frac{e^{-\frac{c\left(x_{n}-y_{n}\right)^{2}}{t}}}{\sqrt{t}} \quad \frac{e^{-\frac{c\left|x^{\prime}-y^{\prime}\right|^{2}}{t}}}{t^{(n-1) / 2}} \quad d y^{\prime} \leq \frac{C}{\sqrt{t}} .
$$

By partitioning $S$ into a finite number of domains, each locally diffeomorphic to a plane region, we find that the above estimate is valid in general.

Later on we shall need to work with the function

$$
E(x)=\int_{0}^{T} B(x, s) A(x, T-s) d s=\int_{0}^{T} w_{t}(x, s) A(x, T-s) d s .
$$

Lemma 1.4. The function $E$ satisfies:

$$
\begin{aligned}
& 0 \leq E(x) \leq C(T), \\
& \int_{\Omega}|\nabla E(x)| \leq C(T) .
\end{aligned}
$$


Proof. The estimate (1.19) follows from (1.9) and Lemma 1.3. To prove (1.20) we write

$$
\begin{aligned}
D_{x} E= & \int_{0}^{T} D_{x} w_{t}(x, s) \cdot A(x, T-s) d s+\int_{0}^{T} w_{t}(x, s) D_{x} A(x, T-s) d s \\
= & {\left[D_{x} w(x, s) \cdot A(x, T-s)\right]_{0}^{T}+\int_{0}^{T} D_{x} w(x, s) A_{t}(x, T-s) d s } \\
& +\int_{0}^{T} w_{t}(x, s) D_{x} A(x, T-s) d s .
\end{aligned}
$$

By Lemmas 1.1, 1.2, each of the last two integrals is in $L^{1}(\Omega)$ with $L^{1}$-norm bounded by $C(T)$. Since also

$$
\int_{\Omega}\left|\left[D_{x} w(x, s) \cdot A(x, T-s)\right]_{0}^{T}\right|=\int_{\Omega}\left|D_{x} w(x, T)\right| \leq C(T),
$$

the assertion (1.20) follows.

Let $\psi$ be the principal eigenfunction of

$$
\begin{aligned}
& -\Delta \psi+\varphi \psi=\lambda \psi \quad \text { in } \Omega \\
& \psi_{\nu}+a \psi=0 \text { on } \partial \Omega, \\
& \psi>0 \text { in } \Omega, \quad \int_{\Omega} \psi^{2}=1 .
\end{aligned}
$$

Then $\psi$ is the minimizer (up to multiplication by \pm 1 ) and $\lambda$ is the minimum of the problem

$$
\lambda=\min _{\Omega}\left\{\int_{\Omega}\left(\frac{1}{2}|\nabla v|^{2}+\frac{1}{2} \varphi v^{2}\right)+\int_{\partial \Omega} \frac{1}{2} a v^{2}\right\} .
$$

Since $\int_{\Omega} \varphi=\sigma T$, by taking a bounded function $v$ in (1.22) we deduce that

$$
\lambda \leq C(T) .
$$

It then follows that

$$
\int_{\Omega}|\nabla \psi|^{2} \leq C(T)
$$


LEMMA 1.5. There holds:

$$
\psi(x) \leq C(T) .
$$

Proof. By (1.24) and Sobolev's inequality,

$$
\int_{\Omega}|\psi|^{q} \leq C(T), \quad q=\frac{2 n}{n-2},
$$

if $n \geq 3$; if $n=2$ then $q$ is any positive number and if $n=1$ then (1.25) holds. Without loss of generality we may assume that $n \geq 2$. We shall compare $\psi$ with the solution $\chi$ to

$$
\begin{aligned}
-\Delta \chi=\lambda \psi & \text { in } \quad \Omega, \\
\chi_{\nu}+a \chi=0 & \text { on } \partial \Omega .
\end{aligned}
$$

Since $-\Delta \psi \leq \lambda \psi$, we deduce that

$$
0 \leq \psi \leq \chi .
$$

Applying $L^{p}$-elliptic estimates to $\chi$ and recalling (1.26), we get

$$
|\chi|_{W^{2, q}} \leq C(T) .
$$

It follows by Sobolev's imbedding that

$$
|\chi|_{L^{q_{1}}(\Omega)} \leq C(T)
$$

where

$$
q_{1}=\left\{\begin{array}{ccc}
\frac{n q}{n-2 q} & \text { if } & q<\frac{n}{2} \\
\infty & \text { if } & q>\frac{n}{2}
\end{array}\right.
$$

and $q_{1}$ is any positive number if $q=\frac{n}{2}$. Consequently

$$
|\psi|_{L^{q_{1}}(\Omega)} \leq C(T) .
$$

If $q_{1}<\infty$ then we can repeat the above process and deduce that

$$
|\psi|_{L^{q_{2}}} \leq C(T)
$$

with

$$
q_{2}=\left\{\begin{array}{ccc}
\frac{n q_{1}}{n-2 q_{1}} & \text { if } & q_{1}<\frac{n}{2} \\
\infty & \text { if } & q_{1}>\frac{n}{2}
\end{array}\right.
$$

and $q_{2}$ any positive number if $q_{1}=\frac{n}{2}$. Since the sequence $q_{1}, q_{2}, \ldots, q_{m}$ cannot remain finite if $m$ is large enough, the assertion (1.25) follows. 
Lemma 1.6. The function $B$ satisfies:

$$
\int_{\Omega} B(x, T) d x \geq c>0
$$

where $c$ is a constant depending on $T$, but otherwise independent of $\varphi$.

The proof will exploit Lemma 1.5 and the following three lemmas.

LEMMA 1.7. If $u>0$ in $\Omega$ and

$$
\int_{\Omega} u^{2}=1, \int_{\Omega} u^{p} \leq C_{1} \text { for some } p>2,
$$

then there exist positive constants $\alpha, b$ depending only on $C_{1}$ and $\Omega$ such that

$$
\operatorname{meas}\{u(x)>\alpha, x \in \Omega\}>b .
$$

Proof. Writing

$$
\begin{gathered}
1=\int_{\{u<\alpha\}} u^{2}+\int_{\{u>\alpha\}} u^{2}<\alpha^{2}|\Omega|+\left\{\int_{\{u>\alpha\}} u^{p}\right\}^{\frac{2}{p}}\{\operatorname{meas}\{u>\alpha\}\}^{\frac{1}{q}} \\
<\alpha^{2}|\Omega|+C_{1}^{\frac{2}{p}}\{\operatorname{meas}\{u>\alpha\}\}^{\frac{1}{q}}
\end{gathered}
$$

where $1 / q+2 / p=1,(1.28)$ follows by choosing $\alpha^{2}|\Omega|=1 / 2$.

LEMMA 1.8. The function $\psi$ satisfies:

$$
\int_{\Omega}|\nabla(\log \psi)|^{2} \leq C(T) .
$$

Proof. Multiplying the equation for $\psi$ by $1 /(\psi+\varepsilon)(\varepsilon>0)$ and integrating over $\Omega$, we get

$$
\begin{array}{r}
\int_{\Omega}(\lambda-\varphi) \frac{\psi}{\psi+\varepsilon}=-\int_{\Omega} \frac{\Delta \psi}{\psi+\varepsilon}=-\int_{\partial \Omega} \psi_{\nu} \frac{1}{\psi+\varepsilon}+\int_{\Omega} \nabla \psi \cdot \nabla \frac{1}{\psi+\varepsilon} \\
=\int_{\partial \Omega} \frac{a \psi}{\psi+\varepsilon}-\int_{\Omega} \frac{|\nabla \psi|^{2}}{(\psi+\varepsilon)^{2}}=\int_{\partial \Omega} \frac{a \psi}{\psi+\varepsilon}-\int_{\Omega}|\nabla \log (\psi+\varepsilon)|^{2} .
\end{array}
$$

Letting $\varepsilon \rightarrow 0$ we find that

$$
\int_{\Omega}|\nabla(\log \psi)|^{2}=\int_{\partial \Omega} a+\int_{\Omega}(\varphi-\lambda) \leq C(T) .
$$


LEMma 1.9. The function $\psi$ satisfies:

$$
\int_{S} \psi(x) d S_{x} \geq e^{-C(T)} .
$$

Proof. By Lemma 1.7 applied to $\psi$, if $\Omega_{0}$ is a compact subdomain of $\Omega$ such that $\operatorname{meas}\left(\Omega \backslash \Omega_{0}\right)<b / 2$, then

$$
\text { meas } \Omega_{*}>\frac{b}{2} \quad \text { where } \quad \Omega_{*}=\left\{x \in \Omega_{0}, \psi(x)>\alpha\right\} .
$$

In the sequel we choose $\Omega_{0}$ to have smooth boundary.

Take a plane $\Pi$ tangent to $S$ at some interior point $x_{0}$ of $S$, and move it in the direction of the inner normal at $x_{0}$. We denote by $\Pi_{\tau}$ the position of $\Pi$ at distant $\tau$ from $x_{0}$. Since

$$
\int \operatorname{meas}\left(\Pi_{\tau} \cap \Omega_{*}\right) d \tau>\frac{b}{2}
$$

there exists a $\tau_{0}$ such that

$$
\operatorname{meas}\left(\Pi_{\tau_{0}} \cap \Omega_{*}\right)>\frac{b}{2 R} \quad(R=\text { diameter of } \Omega) .
$$

We now connect an $S$-neighborhood $S_{0}$ of $x_{0}$ to $\Pi_{\tau_{0}} \cap \Omega$ by smooth curves $l_{\zeta}\left(\zeta \in S_{0}\right)$ such that at each point $(\zeta, s)$ on $l_{\zeta} \quad\left(s\right.$ is the length parameter on $\left.l_{\zeta}\right)$

$$
\frac{1}{C} d S_{\zeta} d s \leq d x \leq C d S_{\zeta} d s .
$$

Denote by $S_{1}$ the subset of points of $S_{0}$ for which $l_{\zeta}$ meets $\Pi_{\tau_{0}} \cap \Omega_{*}$.

Integrating $\log \psi$ along the curves $l_{\zeta}$ with $\zeta \in S_{1}$ and using Jensen's inequality, we get

$$
\begin{aligned}
& \log \int_{S_{1}} \psi(x) \frac{d S_{x}}{\left|S_{1}\right|} \geq \int_{S_{1}} \log \psi(x) \frac{d S_{x}}{\left|S_{1}\right|} \\
& =\frac{1}{\left|S_{1}\right|}\left(\int_{\Pi_{\tau_{0}} \cap \Omega_{*}} \log \psi+\iint \frac{\partial}{\partial l_{\zeta}} \log \psi\right) .
\end{aligned}
$$

The double integral in (1.32) is taken over the set generated by $\left\{l_{\zeta}, \zeta \in S_{1}\right\}$. By Lemma 1.8 and (1.31),

$$
\left|\iint \frac{\partial}{\partial l_{\zeta}} \log \psi\right| \leq C \int_{\Omega}|\nabla \log \psi| \leq C(T) .
$$


Since also $\log \psi>\log \alpha$ on $\Pi_{\tau_{0}} \cap \Omega_{*}$, (1.32) yields

$$
\log \int_{S_{1}} \psi \geq-C(T) .
$$

Therefore also $\log \int_{S} \psi \geq-C(T)$, and the lemma follows.

Proof of Lemma 1.6. Consider the function

$$
g(t)=\int_{\Omega} B(x, t) \psi(x) d x
$$

Clearly

$$
\begin{aligned}
g^{\prime}(t) & =\int_{\Omega} B_{t} \psi=\int_{\Omega}(\Delta B-\varphi B) \psi \\
& =\int_{\Omega}(\Delta \psi-\varphi \psi) B=-\int_{\Omega} \lambda \psi B=-\lambda g,
\end{aligned}
$$

so that

$$
g(T) \geq e^{-\lambda T} g(0) .
$$

But

$$
\begin{aligned}
g(0) & =\int_{\Omega} \int_{S} G(x, 0 ; \eta) d S_{\eta} \psi(x) d x \\
& =\int_{\Omega} \int_{S} \delta_{\eta}(x) \psi(x) d x=\int_{S} \psi(\eta) \geq e^{-C(T)}
\end{aligned}
$$

by Lemma 1.9 , whereas

$$
g(T)=\int_{\Omega} B(x, T) \psi(x) d x \leq C \int_{\Omega} B(x, T) d x
$$

by Lemma 1.5. Hence (1.33) yields the assertion (1.27).

We conclude this section by studying the function

$$
\Psi(x)=\frac{\int_{0}^{T} B(x, s) A(x, T-s) d s}{\int_{\Omega} B(y, T) d y} .
$$


LEMMA 1.10. The function $\Psi$ satisfies:

$$
\int_{\Omega} \Psi(x) d x=T .
$$

Proof. By the semigroup property (or by uniqueness of solutions of (1.2)-(1.4)) and by the symmetry of $G$,

$$
\int_{\Omega} G(x, s ; \eta) G(\xi, T-s ; x) d x=G(\xi, T ; \eta) .
$$

Integrating with respect to $\eta \in S$ and $\xi \in \Omega$, we find that

$$
\int_{\Omega} B(x, s) A(x, T-s) d x=\int_{\Omega} B(\xi, T) d \xi
$$

from which (1.35) follows.

Lemma 1.11. The function $\Psi$ satisfies:

$$
\begin{aligned}
& 0 \leq \Psi \leq C(T), \\
& \int_{\Omega}|\nabla \Psi(x)| d x \leq C(T) .
\end{aligned}
$$

Indeed, this follows immediately from Lemmas 1.4, 1.6.

$\S 2$. Existence in case $\sigma>0$. In this section we establish the existence of a solution to (0.1)-(0.3), (0.5) in case $\sigma>0$. For convenience we replace $\sigma \varphi$ by $\varphi$. Then the system becomes

$$
\begin{aligned}
& \left(\frac{\partial}{\partial t}-\Delta+\varphi(x)\right) G\left(x, t ; x^{\prime}\right)=0, \quad(x, t) \in \Omega_{T}, \\
& \left(\partial_{\nu}+a(x)\right) G\left(x, t ; x^{\prime}\right)=0, \quad x \in \partial \Omega, 0<t<T, \\
& G\left(x, 0 ; x^{\prime}\right)=\delta_{x^{\prime}}(x), \quad x \in \Omega
\end{aligned}
$$

when $\varphi(x)$ is defined by

$$
\begin{aligned}
\varphi & =\sigma \Psi \\
\Psi(x) & =\frac{\int_{0}^{T} d s \int_{S} G(x, s ; \eta) d S_{\eta} \int_{\Omega} G(\xi, T-s ; x) d \xi}{\int_{S} d S_{\eta} \int_{\Omega} G(\xi, T ; \eta) d \xi}
\end{aligned}
$$


THEOREM 2.1. Let $\sigma>0$. Then there exists a solution of (2.1)-(2.4) satisfying:

$$
\varphi \geq 0, \quad \int_{\Omega} \varphi=T, \quad \varphi \in C(\bar{\Omega}), \quad \nabla \varphi \in L^{1}(\Omega) .
$$

Proof. Fix any $1<p<\infty$ and introduce the set, in $L^{p}(\Omega)$ :

$$
\mathcal{A}=\left\{\varphi \in L^{p}(\Omega), \quad|\varphi|_{L^{p}(\Omega)} \leq C^{*}, \quad \varphi \geq 0, \quad \int_{\Omega} \varphi=T\right\},
$$

where $C^{*}$ is a positive constant to be determined.

For any $\varphi \in \mathcal{A}$ we can solve (2.1)-(2.3) as in $\S 1$ and define a mapping $\tilde{\varphi}=W \varphi$ where $\tilde{\varphi}=\Psi, \Psi$ as in (1.34). We need to prove that $\sigma W$ has a fixed point $\varphi$ and that $\varphi \in C(\bar{\Omega}), \nabla \varphi \in L^{1}(\Omega)$.

We first notice that, by Lemma 1.10, 1.11,

$$
\sigma W \quad \text { maps } \mathcal{A} \text { into itself }
$$

provided $C^{*}$ is chosen large enough (depending only on $T$ ).

We next show that

$$
\sigma W(\mathcal{A}) \text { is a compact subset of } \mathcal{A}
$$

where $\mathcal{A}$ is endowed with the $L^{p}(\Omega)$-norm. Indeed, take a sequence $\varphi_{j}$ in $\mathcal{A}$ and set $\tilde{\varphi}_{j}=W \varphi_{j}$. Then

$$
\left|\tilde{\varphi}_{j}\right|_{W^{1,1}} \leq C(T), \quad \text { by Lemma } 1.11 \text {. }
$$

Since $W^{1,1}$ is compactly imbedded in $L^{q}, 1<q<\frac{n}{n-1}$, there exists a subsequence $\varphi_{j}$ such that

$$
\left|\tilde{\varphi}_{j}-\hat{\psi}\right|_{L^{q}} \rightarrow 0, \quad \hat{\psi} \in L^{q}(\Omega) .
$$

By Lemma 1.11 we have $0 \leq \psi_{j} \leq C(T)$, and the same must also hold for $\hat{\psi}$. If we choose $1<q<p$ then, by interpolation,

$$
\begin{aligned}
\left|\tilde{\varphi}_{j}-\hat{\psi}\right|_{L^{p}} \leq C\left|\tilde{\varphi}_{j}-\hat{\psi}\right|_{L^{q}}^{\mu}\left|\tilde{\varphi}_{j}-\hat{\psi}\right|_{L^{\infty}}^{1-\mu} \\
\leq C(T)\left|\tilde{\varphi}_{j}-\hat{\psi}\right|_{L^{q}}^{\mu} \rightarrow 0 \text { if } \quad j \rightarrow \infty
\end{aligned}
$$

(for some $0<\mu<1$ ), which implies the asserted compactness.

We finally claim that

$$
\sigma W \text { is a continuous mapping, }
$$


that is,

$$
\text { if }\left|\varphi_{j}-\varphi\right|_{L^{p}} \rightarrow 0 \text { then }\left|\tilde{\varphi}_{j}-\tilde{\varphi}\right|_{L^{p}} \rightarrow 0
$$

where $\tilde{\varphi}_{j}=W \varphi_{j}, \quad \tilde{\varphi}=W \varphi$.

Denote by $A_{j}, B_{j}, w_{j}$ the functions $A, B, w$ corresponding to $\varphi_{j}$. Then by Lemmas 1.1, 1.2

$$
\begin{aligned}
& \iint_{\Omega_{T}} A_{j, t}^{2}+\int_{\Omega}\left|\nabla A_{j}(x, t)\right|^{2} \leq C, \\
& \iint_{\Omega_{T}} w_{j, t}^{2}+\int_{\Omega}\left|\nabla w_{j}(x, t)\right|^{2} \leq C .
\end{aligned}
$$

It is also clear by uniqueness of solutions to (1.2)-(1.4) that

$$
A_{j} \rightarrow A \quad \text { and } \quad B_{j} \rightarrow B
$$

in the appropriate weak sense. It follows that

$$
\int_{0}^{T} B_{j}(x, s) A_{j}(x, T-s) d s \rightarrow \int_{0}^{T} B(x, s) A(x, T-s) d s
$$

in $L^{1}(\Omega)$ and

$$
\int_{\Omega} B_{j}(x, T) d x \rightarrow \int_{\Omega} B(x, T) d x
$$

Hence $\tilde{\varphi}_{j} \rightarrow \tilde{\varphi}$ in $L^{1}$. Since further

$$
\left|\tilde{\varphi}_{j}\right|_{L^{\infty}} \leq C(T),
$$

we can use interpolation as before to deduce that

$$
\left|\tilde{\varphi}_{j}-\tilde{\varphi}\right|_{L^{p}} \rightarrow 0 \text {. }
$$

Having proved that $\sigma W$ satisfies (2.6), (2.7) and (2.8), we appeal to the Schauder fixed point theorem and deduce that $\sigma W$ has a fixed point $\varphi$. It remains to show that $\varphi \in C(\bar{\Omega})$.

Since $\varphi \in L^{\infty}(\Omega), A_{t}$ and $D_{x} A$ belong to $L^{p}\left(\Omega_{T}\right)$ for any $p<\infty$. Similarly $B_{t}$ and $D_{x} B$ belong to $L^{p}\left(\Omega_{T} \backslash \Omega_{\varepsilon}\right)$ for any $p<\infty, \varepsilon>0$. Also, by Lemma 1.3

$$
\int_{0}^{\tau} B(x, s) d s \leq C \sqrt{\tau} .
$$

It follows that the integral in (1.18) is convergent (at $s=0$ ) uniformly in $x$, and the integrand is continuous (or even Hölder continuous) in $x$ for $0<s \leq T$. Hence $E(x)$ is continuous in $\bar{\Omega}$, and the same is true of $\varphi(x)$. 
REMARK 2.1. Using the form $G=\Gamma+h$ of $G$ where $h$ has weaker singularity than $\Gamma$, one can actually prove that $\varphi \in C^{\alpha}(\bar{\Omega})$ for any $0<\alpha<\frac{1}{2}$.

We shall now establish further the regularity of $\varphi(x)$ :

Theorem 2.2. For any solution of (2.1)-(2.4) with $\varphi \in L^{\infty}(\Omega)$,

$$
\varphi \in C^{\infty}(\Omega)
$$

Proof. Since $\varphi \in L^{\infty}(\Omega)$, we can apply $L^{p}$ estimates to solutions of

$$
u_{t}-\Delta u+\varphi(x) u=0 \text { in } \quad \Omega_{T} .
$$

Recalling that $A(x, t), B(x, t)$ are solutions satisfying

$$
A(x, 0)=1, B(x, 0)=0 \quad \text { if } \quad x \in \Omega,
$$

it follows that

$$
D_{t} A, \nabla_{x} A, D_{t} B \text { and } \nabla_{x} B
$$

belong to $L^{p}\left(Q_{0}\right)$ for any $1<p<\infty$ and for any compact subdomain $Q_{0}$ of

$$
\Omega_{T} \cup\{\Omega \times(t=0)\} \cup\{\Omega \times(t=T)\} .
$$

Hence $A$ and $B$ belong to $C^{\alpha}\left(Q_{0}\right)$ and, by (2.4), $\varphi \in C^{\alpha}(\Omega)$.

We can now apply the Schauder estimates to (2.10) and conclude that the functions in (2.11) belong to $C^{2+\alpha}\left(Q_{0}\right), Q_{0}$ as before. It follows that $\varphi \in C^{2+\alpha}(\Omega)$. Repeating the last argument step-by-step it follows that $\varphi \in C^{2 m+\alpha}$ for all positive integers $m$.

REMARK 2.2. If $\sigma$ is small then the mapping $\sigma W$ is a contraction and, consequently, the solution is unique.

REMARK 2.3. Theorem 2.1 extends to the boundary condition

$$
u=0 \quad \text { on } \quad \partial \Omega \times(0, T),
$$

but one must replace

$$
\int_{S} G(x, s ; \eta) d S_{\eta}
$$


by

$$
\int_{S^{\delta}} G(x, s ; \eta) d S_{\eta}
$$

where $S^{\delta}$ is obtained from $S$ by moving along the inner normals a distance $\delta ; \delta$ is positive and small. To explain this notice that if $\varphi$ is any "reasonable" function and $G$ is the Green function for (2.1) with the boundary condition (2.12), then $G(x, s ; \eta)=0$ for any $\eta \in \partial \Omega$ so that the integral in (2.13) vanishes. Thus there is clearly a mathematical need to modify (2.13) in the definition of $\varphi$ as given in (0.3). The reason why (2.14) should be the correct replacement for (2.13) is the following: Each polymer chain is terminally attached to $S$, that is, the first Kuhn segment is fixed. Thus the probabilistic considerations of the SCF theory should disregard the first segment. This means that (2.13) should be replaced by (2.14) where $\delta$ is the Kuhn length.

One can check that Theorem 2.1 extends to the present boundary condition (2.12); the main difference occurs in the proof of Lemma 1.6 or, more specifically, in the proof of the analog of Lemma 1.9,

$$
\int_{S^{\delta}} \psi(x) d S_{x} \geq e^{-C(T)} .
$$

To prove (2.15) we can proceed essentially as before provided we can establish the following version of Lemma 1.8:

$$
\int_{\Omega}|\nabla(\log \psi)|^{2} \zeta^{2} \leq C(T)
$$

where $\zeta$ is any function in $C_{0}^{\infty}(\Omega)$.

Proof of (2.16). Multiplying the equation for $\psi$ by $\zeta^{2} / \psi$ and integrating over $\Omega$, we get

$$
\begin{gathered}
\int_{\Omega}(\varphi-\lambda) \zeta^{2}=\int_{\Omega} \frac{\Delta \psi}{\psi} \zeta^{2}=-\int_{\Omega} \nabla \psi \cdot \nabla\left(\frac{\zeta^{2}}{\psi}\right) \\
=\int_{\Omega} \zeta^{2} \nabla \psi \cdot \frac{\nabla \psi}{\psi^{2}}-2 \int \frac{\nabla \psi}{\psi} \zeta \cdot \nabla \zeta \\
\geq \frac{1}{2} \int_{\Omega} \zeta^{2} \frac{|\nabla \psi|^{2}}{\psi^{2}}-C \int_{\Omega}|\nabla \zeta|^{2},
\end{gathered}
$$

and (2.16) follows.

Theorem 2.2 also extends to the boundary condition (2.12). 
§3. The case $\sigma<0$. In this case $G$ satisfies

$$
\left(\frac{\partial}{\partial t}-\Delta-\varphi(x)\right) G\left(x, t ; x^{\prime}\right)=0, \quad(x, t) \in \Omega_{T}
$$

and, otherwise, (2.2)-(2.4) remain the same with $\varphi=-\sigma \Psi=|\sigma| \Psi$ in (2.4). Similarly, $A$ and $B$ satisfy the equation

$$
u_{t}-\Delta u-\varphi(x) u=0
$$

and otherwise are defined as before; for simplicity we shall designate from now on $|\sigma|$ by $\sigma$, so that our system becomes $(3.2),(2.2)-(2.4)$ with $\sigma>0$.

THEOREM 3.1. If $n=1$, then there exists a solution of (3.1), (2.2)-(2.4) (with $\sigma$ positive in (2.4)) satisfying:

$$
\varphi \geq 0, \int_{\Omega} \varphi=T, \varphi \in C(\bar{\Omega}), \nabla \varphi \in L^{1}(\Omega)
$$

and $\varphi \in C^{\infty}(\Omega)$.

Proof. Since $n=1$, for any $\varepsilon>0$

$$
\left|v^{2}\right|_{L^{\infty}(\Omega)}<\varepsilon \int_{\Omega}|\nabla v|^{2}+C_{\varepsilon} \int_{\Omega} v^{2}
$$

Since $\int \varphi=T$, this implies that

$$
\frac{1}{4} \int_{\Omega}|\nabla v|^{2}-\int_{\Omega} \varphi v^{2} \geq-C(T) \int_{\Omega} v^{2} .
$$

Analogously to (1.12) we have

$$
\iint_{\Omega_{t}} A_{t}^{2}+\frac{1}{2} \int_{\Omega}|\nabla A(x, t)|^{2} \leq \iint_{\Omega_{t}} \varphi A^{2}+C(T) .
$$

Using (3.5) to estimate the right-hand side, and using also the relation

$$
A(x, t)=1+\int_{0}^{t} A_{t}(x, s) d s
$$


one can easily derive, by Gronwall's inequality, the bound

$$
\iint_{\Omega_{T}} A_{t}^{2}+\int_{\Omega}|\nabla A(x, t)|^{2} d x+\int_{\Omega} A^{2}(x, t) d x \leq C(T) .
$$

From Sobolev's imbedding we then also get

$$
0 \leq A(x, t) \leq C(T) .
$$

We next consider $B(x, t)$, or $w(x, t)$. Using (3.5) and proceeding analogously to (1.15), we get

$$
\iint_{\Omega_{T}} w_{t}^{2}+\int_{\Omega}|\nabla w(x, t)|^{2} d x \leq C(T)+C(T) \int_{\Omega} w^{2}(x, t) d x .
$$

Using the relations

$$
\begin{aligned}
\int_{\Omega} w^{2}(x, t) d x & =\iint_{\Omega_{t}} 2 w w_{t}(x, t) d x \\
& \leq 2 \iint_{\Omega_{T}} w^{2}+\frac{1}{2} \iint w_{t}^{2}
\end{aligned}
$$

to estimate the right-hand side of (3.8), we then obtain, by Gronwall's inequality,

$$
\iint_{\Omega_{t}} w_{t}^{2}+\int_{\Omega}|\nabla w(x, t)|^{2} d x \leq C(T) .
$$

The estimates (3.6), (3.7) and (3.9) replace Lemmas 1.1 and 1.2. From these estimate we conclude, as in $\S 1$, that

$$
\int_{\Omega}|\nabla E(x)| \leq C(T) .
$$

Since also $\int_{\Omega} E(x) \leq C(T)$ and $n=1$, it follows that

$$
0 \leq E(x) \leq C(T)
$$

thus Lemma 1.4 is also valid.

We next need an analog of Lemma 1.6. But this is very easy: since $G \geq \Gamma$ (by comparison),

$$
\int_{\Omega} B(x, T) d x \geq \int_{S} \int_{\Omega} \Gamma\left(x, t ; x^{\prime}\right) d x d S_{x^{\prime}} \geq C(T)>0 ;
$$

of course, since $n=1, S^{\prime}$ consists of just one or the two endpoints of $\Omega$.

With the above estimates on $E$ and $\int B(x, T) d x$, we can repeat the proof of Theorem 2.1, and then also of Theorem 2.2, and thus establish Theorem 3.1. 
REMARK 3.1. If $\sigma$ is sufficiently small then one can establish existence and uniqueness for (3.1), (2.2)-(2.4) for any $n \geq 1$. Indeed, we define

$$
\mathcal{A}=\left\{\varphi \in L^{\infty}(\Omega), 0 \leq \varphi \leq M, \int_{\Omega} \varphi=T\right\}, M>T /|\Omega|
$$

Then the proofs of Lemma 1.1, 1.2 remain valid for our present equation (3.2) with constants $C(M)$ which depend on $M$. But then also (3.6)-(3.8) hold with $C=C(M)$, and the same for $(3.10),(3.11)$. Since $(3.12)$ is also valid, $W$ is a bounded operator from $\mathcal{A}$ into $\mathcal{A}$ (with the $L^{\infty}$-norm), and $\sigma W$ is then a contraction.

REMARK 3.2. It is not clear whether uniqueness is true in case $\sigma>0$. For $\sigma$ negative and large in absolute value, numerical calculations show non-uniqueness when the Laplacian is discretized and the number of interior partition points is 2 or 3 .

Acknowledgement. We would like to thank Mathew Tirrell for introducing us to the topic studied in this paper.

\section{REFERENCES}

[1] P.-G. DE Gennes, Some conformation problems for long macromolecular, Rep. Prog. Phys., 32 (1969), 187-205.

[2] A. Dolan and S.F. Edwards, Theory of the stabilization of colloids by adsorbed polymer, Proc. Roy. Soc. London, A. 337 (1974), 509-516.

[3] A. Dolan and S.F. Edwards, The effect of excluded volume on polymer dispersant action, Proc. Royal Soc. London, A. 343 (1975), 427-442.

[4] M. Muthukumar and J.S. Ho, Self-consistent field theory of surfaces with terminally attached chains, Macromolecules, 22 (1989), 965-973.

[5] S.S. Patel, Self-consistent field theory of terminally attached polymers: Dolan-Edwards revisited, submitted to Macromolecules.

[6] H.J. Ploenn and W.B. Russel, Interaction between colloidal particles and soluble polymer, submitted to Advances in Chemical Engineering. 
Werner A. Stahel, Robust Statistics: From an Intellectual Game to a Consumer Product

Avner Friedman and Fernando Reitich, The Stefan Problem with Small Surface Tension

E.G. Kalnins and W. Miller, Jr., Separation of Variables Methods for Systems of Differential Equations in Mathematical Physics

Mitchell Luskin and George R. Sell, The Construction of Inertial Manifolds for Reaction-Diffusion Equations by Elliptic Regularization

Konstantin Mischaikow, Dynamic Phase Transitions: A Connection Matrix Approach

Philippe Le Floch and Li Tatsien, A Global Asymptotic Expansion for the Solution to the Generalized Riemann Problem

Matthew Witten, Ph.D., Computational Biology: An Overview

Matthew Witten, Ph.D., Peering Inside Living Systems: Physiology in a Supercomputer

Michael Renardy, An existence theorem for model equations resulting from kinetic theories of polymer solutions

Daniel D. Joseph and Luigi Preziosi, Reviews of Modern Physics: Addendum to the Paper "Heat Waves"

Luigi Preziosi, An Invariance Property for the Propagation of Heat and Shear Waves

Gregory M. Constantine and John Bryant, Sequencing of Experiments for Linear and Quadratic Time Effects

Prabir Daripa, On the Computation of the Beltrami Equation in the Complex Plane

Philippe Le Floch, Shock Waves for Nonlinear Hyperbolic Systems in Nonconservative Form

A.L. Gorin, D.B. Roe and A.G. Greenberg, On the Complexity of Pattern Recognition Algorithms On a Tree-Structured Parallel Computer

Mark J. Friedman and Eusebius J. Doedel, Numerical computation and continuation of invariant manifolds connecting fixed points

Scott J. Spector, Linear Deformations as Global Minimizers in Nonlinear Elasticity

Denis Serre, Richness and the classification of quasilinear hyperbolic systems

L. Preziosi and F. Rosso, On the stability of the shearing flow between pipes

Avner Friedman and Wenxiong Liu, A system of partial differential equations arising in electrophotography

Jonathan Bell, Avner Friedman, and Andrew A. Lacey, On solutions to a quasilinear diffusion problem from the study of soft tissue

601 David G. Schaeffer and Michael Shearer, Loss of hyperbolicity in yield vertex plasticity models under nonproportional loading

Herbert C. Kranzer and Barbara Lee Keyfitz, A strictly hyperbolic system of conservation laws admitting singular shocks

S. Laederich and M. Levi, Qualitative dynamics of planar chains

Milan Miklavčič, A sharp condition for existence of an inertial manifold

Charles Collins, David Kinderlehrer, and Mitchell Luskin, Numerical approximation of the solution of a variational problem with a double well potential

Todd Arbogast, Two-phase incompressible flow in a porous medium with various nonhomogeneous boundary conditions

Peter Poláčik, Complicated dynamics in scalar semilinear parabolic equations in higher space dimension

Bei Hu, Diffusion of penetrant in a polymer: a free boundary problem

Mohamed Sami ElBialy, On the smoothness of the linearization of vector fields near resonant hyperbolic rest points

Max Jodeit, Jr. and Peter J. Olver, On the equation $\operatorname{grad} f=M \operatorname{grad} g$

Shui-Nee Chow, Kening Lu, and Yun-Qiu Shen, Normal form and linearization for quasiperiodic systems

Prabir Daripa, Theory of one dimensional adaptive grid generation

Michael C. Mackey and John G. Milton, Feedback, delays and the origin of blood cell dynamics

D.G. Aronson and S. Kamin, Disappearance of phase in the Stefan problem: one space dimension

Martin Krupa, Bifurcations of relative equilibria
D.D. Joseph, P. Singh, and K. Chen, Couette flows, rollers, emulsions, tall Taylor cells, phase separation and inversion, and a chaotic bubble in Taylor-Couette flow of two immiscible liquids

Artemio González-López, Niky Kamran, and Peter J. Olver, Lie algebras of differential operators in two complex variables 
conservation laws

Xinfu Chen, Axially symmetric jets of compressible fluid

J. David Logan, Wave propagation in a qualitative model of combustion under equilibrium conditions

M.L. Zeeman, Hopf bifurcations in competitive three-dimensional Lotka-Volterra Systems

Allan P. Fordy, Isospectral flows: their Hamiltonian structures, Miura maps and master symmetries

Daniel D. Joseph, John Nelson, Michael Renardy, and Yuriko Renardy, Two-Dimensional cusped interfaces

Avner Friedman and Bei Hu, A free boundary problem arising in electrophotography

Hamid Bellout, Avner Friedman and Victor Isakov, Stability for an inverse problem in potential theory

Barbara Lee Keyfitz, Shocks near the sonic line: A comparison between steady and unsteady models for change of type

Barbara Lee Keyfitz and Gerald G. Warnecke, The existence of viscous profiles and admissibility for transonic shocks

P. Szmolyan, Transversal heteroclinic and homoclinic orbits in singular perturbation problems

Philip Boyland, Rotation sets and monotone periodic orbits for annulus homeomorphisms

Kenneth R. Meyer, Apollonius coordinates, the N-body problem and continuation of periodic solutions

Chjan C. Lim, On the Poincare-Whitney circuitspace and other properties of an incidence matrix for binary trees

K.L. Cooke and I. Györi, Numerical approximation of the solutions of delay differential equations on an infinite interval using piecewise constant arguments

Stanley Minkowitz and Matthew Witten, Periodicity in cell proliferation using an asynchronous cell population

M. Chipot and G. Dal Maso, Relaxed shape optimization: The case of nonnegative data for the Dirichlet problem

Jeffery M. Franke and Harlan W. Stech, Extensions of an algorithm for the analysis of nongeneric Hopf bifurcations, with applications to delay-difference equations

Xinfu Chen, Generation and propagation of the interface for reaction-diffusion equations

Philip Korman, Dynamics of the Lotka-Volterra systems with diffusion

Harlan W. Stech, Generic Hopf bifurcation in a class of integro-differential equations

Stephane Laederich, Periodic solutions of non linear differential difference equations

Peter J. Olver, Canonical Forms and Integrability of BiHamiltonian Systems

S.A. van Gils, M.P. Krupa and W.F. Langford, Hopf bifurcation with nonsemisimple 1:1 Resonance

R.D. James and D. Kinderlehrer, Frustration in ferromagnetic materials

Carlos Rocha, Properties of the attractor of a scalar parabolic P.D.E.

Debra Lewis, Lagrangian block diagonalization

Richard C. Churchill and David L. Rod, On the determination of Ziglin monodromy groups

Xinfu Chen and Avner Friedman, A nonlocal diffusion equation arising in terminally attached polymer chains

Peter Gritzmann and Victor Klee, Inner and outer j- Radii of convex bodies in finitedimensional normed spaces

P. Szmolyan, Analysis of a singularly perturbed traveling wave problem

Stanley Reiter and Carl P. Simon, Decentralized dynamic processes for finding equilibrium

Fernando Reitich, Singular solutions of a transmission problem in plane linear elasticity for wedge-shaped regions

Russell A. Johnson, Cantor spectrum for the quasi-periodic Schrödinger equation

Wenxiong Liu, Singular solutions for a convection diffusion equation with absorption

Deborah Brandon and William J. Hrusa, Global existence of smooth shearing motions of a nonlinear viscoelastic fluid

James F. Reineck, The connection matrix in Morse-Smale flows II

Claude Baesens, John Guckenheimer, Seunghwan Kim and Robert Mackay, Simple resonance regions of torus diffeomorphisms

Willard Miller, Jr., Lecture notes in radar/sonar: Topics in Harmonic analysis with applications to radar and sonar

Calvin H. Wilcox, Lecture notes in radar/sonar: Sonar and Radar Echo Structure

Richard E. Blahut, Lecture notes in radar/sonar: Theory of remote surveillance algorithms 